DOI: $10.18027 / 2224-5057-2021-11-3 s 2-30$

Цитирование: Орел Н.Ф., Артамонова Е.В., Горбунова В.А., Делекторская В.В., Емельянова Г.С., Любимова Н.В. и соавт. Практические рекомендации по лекарственному лечению нейроэндокринных неоплазий желудочно-кишечного тракта, поджелудочной железы и других локализаций. Злокачественные опухоли : Практические рекомендации RUSSCO \#3s2, 2021 (том 11). 30

\title{
ПРАКТИЧЕСКИЕ РЕКОМЕНДАЦИИ ПО ЛЕКАРСТВЕННОМУ ЛЕЧЕНИЮ НЕЙРОЭНДОКРИННЫХ НЕОПЛАЗИЙ ЖЕЛУДОЧНО-КИШЕЧНОГО ТРАКТА, ПОДЖЕЛУДОЧНОЙ ЖЕЛЕЗЫ И ДРУГИХ ЛОКАЛИЗАЦИЙ
}

Коллектив авторов: Орел Н.Ф., Артамонова Е.В., Горбунова В.А., Делекторская В.В., Емельянова Г.С., Любимова Н.В., Маркович А.А., Орлова К.В., Орлова Р.В., Трифанов В.С.

Ключевые слова: нейроэндокринные опухоли, поджелудочная железа, желудочно-кишечный тракт, иммунотерапия, гормонотерапия, химиотерапия, аналоги соматостатина, рецепторы соматостатина

\section{1. КЛАССИФИКАЦИЯ И ОПРЕДЕЛЕНИЕ СТАДИИ}

\section{1. По степени злокачественности}

Согласно унифицированной классификации все нейроэндокринные новообразования обозначены термином нейроэндокринные неоплазии (HЭH, NEN), который объединяет высокодифференцированные нейроэндокринные опухоли (НЭО, NET) и низкодифференцированные нейроэндокринные раки или карциномы (НЭР, НЭК, NEC)

Алгоритм выбора лечения нейроэндокринных неоплазий ЖКТ и поджелудочной железы (ПЖ) зависит от типа и распространенности неоплазии и ориентирован на классификацию ВОЗ НЭО ПЖ 2017 г. и ЖКТ 2019 г. (табл. 1). Для НЭО панкреатической локализации в 2017 г. выделена группа G3 (высокодифференцированные НЭО с повышенной пролиферативной активностью) (табл. 2). В 2019 г. такая же классификация принята ВОЗ для НЭО ЖКТ. НЭР ЖКТ и ПЖ подразделяют на мелкоклеточный рак и крупноклеточный нейроэндокринный рак. Также, выделена группа смешанных опухолей MINEN 
Таблица 1. Классификация нейроэндокринных неоплазий (NEN) гастроинтестинальных и органов гепатопанкреатобилиарной системы (BО3, 2019 г.) $)^{1}$

\begin{tabular}{|c|c|c|c|c|}
\hline Терминология & $\begin{array}{l}\text { Степень дифферен- } \\
\text { цировки }\end{array}$ & $\begin{array}{l}\text { Степень злокаче- } \\
\text { ственности (G) }\end{array}$ & Митозы $/ 2$ мм $^{2}$ & Индекс Кі67, \% \\
\hline HЭО (NET) G1 & $\begin{array}{l}\text { Высокодифференциро- } \\
\text { ванные }\end{array}$ & Низкая & $<2$ & $<3$ \\
\hline HЭО (NET) G2 & & Промежуточная & $2-20$ & $3-20$ \\
\hline HЭO (NET) G3 & & Высокая & $>20$ & $>20$ \\
\hline $\begin{array}{l}\text { НЭР (NEC), } \\
\text { мелкоклеточный } \\
\text { тип (SCNEC) }\end{array}$ & $\begin{array}{l}\text { Низкодифференциро- } \\
\text { ванные }\end{array}$ & Высокая & $>20$ & $>20$ \\
\hline $\begin{array}{l}\text { НЭР (NEC), } \\
\text { крупноклеточный } \\
\text { тип (LCNEC) }\end{array}$ & & & $>20$ & $>20$ \\
\hline MINEN & $\begin{array}{l}\text { Высоко- или низко- } \\
\text { дифференцированные }\end{array}$ & Различная & $\begin{array}{l}\text { Различное } \\
\text { чсло }\end{array}$ & Различный \\
\hline
\end{tabular}

1 Нейроэндокринные неоплазии - термин, объединяющий высокодифференцированные НЭО и низкодифференцированный НЭР. Термин MINEN относится к смешанным опухолям, нейроэндокринный компонент которых составляет не менее 30\%. Индекс пролиферации Кi-67 базируется на оценке $\geq 500$ клеток в областях наиболее высокого ядерного мечения (hot spots), митотический индекс - на оценке митозов в 50 полях зрения при большом увеличении (репрезентативное поле зрения 0,2 мм²) в областях наибольшей плотности и выражается как митотический индекс в 10 репрезентативных полях зрения (2 мм²). $G$ определяется на основании наивысшего показателя. Для оценки индекса Кі-67 рекомендуется подсчет с использованием распечатанного изображения.

\section{2. Стадирование по системе TNM}

Определение стадии НЭН осуществляется в соответствии с классификацией TNM для органа, в котором опухоль возникла. В настоящее время в России используется 8-е издание классификации TNM. В 7-ю редакцию руководства по определению стадий злокачественных опухолей AJCC/UICC (2009 г.) НЭО включены впервые и для большинства локализаций содержат особые, специфичные только для них, критерии (НЭО желудка, тонкой кишки, толстой кишки, аппендикса). В настоящее время для НЭО ПЖ в классификацию ВОЗ 2017 г. включены критерии 8-й редакции TNM классификации AJCC/UICC (2016 г.). Классификация TNM для НЭР ПЖ соответствует принципам стадирования протоковой аденокарциномы Пж.

\section{3. Деление по функциональной активности}

- Функционирующие

- Не функционирующие (табл. 9). 


\section{2. ДИАГНОСТИКА}

Диагноз НЭН ЖКТ и ПЖ основывается на данных осмотра, результатах инструментальных методов обследования и патоморфологическом заключении. Первичное обследование должно быть проведено до всех видов лечебных воздействий и включает в себя:

- сбор анамнеза и осмотр; осмотр включает пальпацию лимфоузлов регионарных зон, а также выявление симптомов, подозрительных в отношении отдаленных метастазов или наличия карциноидного синдрома;

- общий анализ крови с подсчетом лейкоцитарной формулы и количества тромбоцитов;

- биохимический анализ крови с определением показателей функции печени, почек, кальция, глюкозы;

- УзИ органов брюшной полости и малого таза, периферических лимфоузлов;

- R-графию органов грудной клетки;

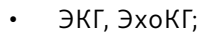

- КТ/МРТ органов брюшной полости и малого таза с в/в контрастированием;

- КТ органов грудной клетки - по показаниям;

- радиоизотопное исследование скелета+R-графию и/или KT/MPT зон накопления РФП - по показаниям;

- биопсию опухоли с патоморфологическим исследованием опухолевой ткани;

- ИГХ исследование с определением в опухолевой ткани экспрессии хромогранина А, синаптофизина, Кi67, рецепторов соматостатина 2 и 5 типа - по показаниям;

- в случае длительного течения заболевания в периоды доказанного прогрессирования рекомендуется повторная биопсия для определения степени злокачественности;

- биохимические маркеры: кровь - хромогранин А, серотонин, панкреатический полипептид (при НЭО ПЖ, ЖКТ и метастазах НЭО не выявленной первичной локализации); гастрин, глюкагон, инсулин, вазоактивный интестинальный пептид, соматостатин, кальцитонин, АКТГ, кортизол - по показаниям; при карциноидном синдроме - 5-ГИУК в суточной моче; ежегодно - NTproBNP;

- К КТ, МРТ головного мозга - по показаниям;

- $\quad$ ПЭТ/КТ с ${ }^{18} \mathrm{~F}-$ ФДГ一 по показаниям (для G2, G3);

- ПЭT/KT с 68DOTA-TATE Ga-DOTA-TOC, DOTA-NOC, (DOTA конъюгаты рецепторов соматостатина) - по показаниям (для G1, G2);

- сцинтиграфия с пентетреодитом и/или ${ }^{99 m T c-Т е к т р о т и д о м ~ п о ~ п о к а з а н и я м ~(д л я ~ G 1, ~ G 2) ; ~}$

- сцинтиграфия c $\ln ^{111}$ (октреоскан) - по показаниям (для G1, G2);

- консультацию медицинского генетика - по показаниям (для больных с синдромом $\mathrm{MЭН).}$ 


\section{3. ЛЕЧЕНИЕ}

\section{1. Лекарственное лечение}

\subsection{1. Гормонотерапия (аналоги соматостатина)}

\subsubsection{1. Гормонотерапия высокодифференцированных нейроэндокринных опухолей (G1, G2)}

Аналоги соматостатина пролонгированного действия (октреотид и ланреотид) как самостоятельное лечение применяются при высокодифференцированных НЭО ЖКТ и ПЖ: октреотид - 30 мг в/м 1 раз в 28 дней, ланреотид - 120 мг п/к 1 раз в 28 дней. Возможно назначение аналогов соматостатина в дозе 30 мг 1 раз в 28 дней в комбинации с ИФН- $\alpha$, цитостатиками, таргетными препаратами для контроля симптомов карциноидного синдрома. В соответствии с инструкцией по использованию пролонгированных форм октреотида лечение начинается с октреотида короткого действия. Октреотид короткого действия применяется п/к в дозе 100 мкг 3 раза в сутки в течение 2 недель перед назначением пролонгированных форм, затем при удовлетворительной переносимости рекомендуется пролонгированный октреотид длительного действия в дозе 30 мг в/м 1 раз в 28 дней. При необходимости возможно увеличение дозы октреотида до 40-60 мг 1 раз в 28 дней или уменьшение интервалов между введениями аналогов соматостатина до 1 раза в 14-21 д ней (табл. 3). Для ланреотида рекомендуемая доза составляет 120 мг п/к каждые 4 недели, при прогрессировании возможно уменьшение интервала между введениями до 2-3 недель или эскалация дозы до 180 мг при наличии дозировки 60 мг или 90 мг. Данное лечение проводится до прогрессирования или непереносимой токсичности. При прогрессировании заболевания дозу аналогов соматостатина необходимо увеличить либо сократить интервалы между введениями, второй компонент комбинированной терапии (таргетный препарат, цитостатик, ИФН- $\alpha$ ) следует отменить и заменить на другое лечение. После завершения курса комбинированного лекарственного лечения аналоги соматостатина назначаются в качестве поддерживающей терапии на длительный срок. Имеются ограниченные данные по применению аналогов соматостатина во время беременности. При необходимости их использования при беременности решение должно приниматься мультидисциплинарной комиссией с участием онколога, эндокринолога и акушера-гинеколога. При непереносимости какого-либо препарата или прогрессировании опухолевого процесса один препарат может быть заменен на другой (октреотид на ланреотид и наоборот).

Таблица 2. Рекомендуемые варианты гормонотерапии высокодифференцированных нейроэндокринных опухолей

\begin{tabular}{|l|l|}
\hline Препарат & Режим применения \\
\hline Октреотид & $\begin{array}{l}100 \text { мкг } 3 \text { раза сут. п/к в течение } 2 \text { нед., далее-переход на депо формы } \\
\text { (в соответствии с инструкцией по использованию пролонгированных } \\
\text { октреотидов лечение начинается с октреотида короткого действия) }\end{array}$ \\
\hline Октреотид пролонгированный & 30 мг в/м 1 раз в 28 дней в качестве противоопухолевого лечения \\
\hline Ланреотид & 120 мг п/к 1 раз в 28 дней \\
\hline
\end{tabular}




\subsubsection{2. Гормонотерапия нейроэндокринного рака}

Аналоги соматостатина пролонгированного действия при нейроэндокринных карциномах используются для контроля симптомов или при наличии экспрессии рецепторов соматостатина в опухоли применяются в комбинации с цитостатиками в дозе 20 мг 1 раз в 28 дней в/м для октреотида и 120 мг 1 раз в 28 дней п/к для ланреотида.

\subsection{2. Таргетная терапия}

\subsubsection{1. Таргетная терапия высокодифференцированных нейроэндокринных опухолей (G1, G2)}

Эверолимус применяется для лечения высокодифференцированных диссеминированных НЭО любых локализаций, а также метастазов НЭО не выявленной первичной локализации. Препарат может использоваться в качестве антипролиферативной терапии во II-III линиях после лечения аналогами соматостатина в последующей комбинации с аналогами соматостатина, а также в I линии как самостоятельный препарат в монотерапии - при отсутствии экспрессии рецепторов к соматостатину в опухолевых о чагах; применяется в дозе 10 мг/сутки внутрь ежедневно до прогрессирования или неприемлемой токсичности; при развитии симптомов токсичности доза может быть снижена до 5 мг/сутки.

Сунитиниб зарегистрирован только для лечения НЭО ПЖ и рекомендуется во ІІ линии после лечения аналогами соматостатина в комбинации с аналогами соматостатина или в I линии лечения при отсутствии рецепторов к соматостатину в монотерапии; применяется в дозе 37,5 мг/сутки (используются таблетки 25 мг и 12,5 мг) внутрь ежедневно до прогрессирования или неприемлемой токсичности. При развитии симптомов токсичности доза может быть уменьшена до 25 мг/сутки.

Оптимальная последовательность назначения таргетных препаратов при НЭО ПЖ четко не определена. В случае прогрессирования на одном из таргетных препаратов рекомендуется использование другого таргетного препарата. Рекомендуемые режимы таргетной терапии НЭО G1, G2 представлены в табл. 3.

Таблица 3. Рекомендуемые режимы таргетной терапии высокодифференцированных нейроэндокринных опухолей $(\mathrm{G} 1, \mathrm{G} 2)$

\begin{tabular}{|l|l|}
\hline Препарат & Режим применения \\
\hline Эверолимус & $\begin{array}{l}\text { 10мг/сут. внутрь ежедневно до прогрессирования или непереносимой } \\
\text { токсичности }\end{array}$ \\
\hline $\begin{array}{l}\text { Сунитиниб (только } \\
\text { для больных с НЭО Пж) }\end{array}$ & $\begin{array}{l}37,5 \text { мг/сут. внутрь ежедневно до прогрессирования или непереносимой } \\
\text { токсичности }\end{array}$ \\
\hline
\end{tabular}

\subsubsection{2. Таргетная терапия нейроэндокринного рака и НЭО G3}

Применение таргетной терапии при НЭР и НЭО G3 не рекомендуется. 


\subsection{3. Иммунотерапия}

\subsubsection{1. Иммунотерапия высокодифференцированных нейроэндокринных опухолей $(\mathrm{G} 1, \mathrm{G} 2)$}

ИФН- $\alpha$ является терапией II линии лечения высокодифференцированных НЭО и рекомендуется как дополнение к терапии аналогами соматостатина при недостаточном контроле симптомов функционирующих опухолей. Кроме того, ИФН- $\alpha$ может использоваться в качестве терапии I линии при отсутствии экспрессии рецепторов соматостатина. Рекомендуемая доза ИФН- $\alpha$ составляет 3 млн. МЕ п/к 3-5 раз в нед. до прогрессирования или непереносимой токсичности.

\subsubsection{2. Иммунотерапия нейроэндокринного рака и НЭО G3}

Рекомендуется использовать ИФН- $\alpha$ как добавление к терапии аналогами соматостатина при недостаточном контроле симптомов функционирующих опухолей (табл. 4).

Таблица 4. Рекомендуемый режим иммунотерапии нейроэндокринных опухолей

\begin{tabular}{|l|l|}
\hline Препарат & Режим применения \\
\hline ИФН- $\alpha$ & 3 млн. МЕ п/к 3-5 раз в нед. до прогрессирования или непереносимой токсичности \\
\hline
\end{tabular}

При мелкоклеточном раке и метастазах мелкоклеточного рака из невыявленного первичного очага возможно использование схем иммунотерапии, зарегистрированных для мелкоклеточного рака легкого.

\subsection{4. Химиотерапия}

\subsubsection{1. Химиотерапия высокодифференцированных нейроэндокринных опухолей (G1, G2, G3)}

Применение XT при НЭО ЖКТ и ПЖ G1 в I линии не рекомендуется за исключением случаев быстрого прогрессирования; в этих случаях возможно назначение XT совместно с аналогами соматостатина. При НЭО G2 применение XТ в 1 линии \pm аналоги соматостатина рекомендовано при значительной массе опухоли и наличии карциноидного синдрома. Рекомендуется использовать режимы, не содержащие цисплатин и карбоплатин (табл. 5). Кроме того, в 2020 г. оригинальный отечественный препарат Араноза (арабинопиранозилметил нитрозомочевина) был зарегистрирован по новому показанию: монотерапия нейроэндокринных опухолей различной локализации. Араноза (арабинопиранозилметил нитрозомочевина) также может использоваться в сочетании с аналогами соматостатина.

Таблица 5. Рекомендуемые режимы химиотерапии НЭО G2-G3

\begin{tabular}{|l|l|l|}
\hline Режим & Схема & Интервал между циклами \\
\hline $\begin{array}{l}\text { Араноза (арабинопирано- } \\
\text { зилметил нитрозомочевина) }\end{array}$ & 500-650 мг/м² в/в струйно 1-3 дни & Каждые 3-4 недели \\
\hline
\end{tabular}




\begin{tabular}{|c|c|c|}
\hline Режим & Схема & Интервал между циклами \\
\hline XELOX & $\begin{array}{l}\text { Оксалиплатин } 130 \text { мг/м² в/в в 1-й день + } \\
\text { капецитабин } 2000 \text { мг/м² внутрь в 1-14-й дни }\end{array}$ & Каждые 3 недели \\
\hline Капецитабин & Капецитабин 2500 мг/м² внутрь в 1-14-й дни & Каждые 3 недели \\
\hline $\begin{array}{l}\text { Капецитабин (метрономный } \\
\text { режим) }\end{array}$ & Капецитабин 500 мг внутрь 2-3 раза в день & Ежедневно \\
\hline TemCap & $\begin{array}{l}\text { Темозоломид } 150 \text { мг/м² внутрь в 10- } \\
\text { 14-й дни + капецитабин } 2000 \text { мг/м² внутрь } \\
\text { в 1-14-й дни }\end{array}$ & Каждые 4 недели \\
\hline $\mathrm{TI}^{2}$ & $\begin{array}{l}\text { Темозоломид 150-200 мг/м²/сут. } \\
\text { внутрь в 1-5-й дни + иринотекан } \\
250 \text { мг/м² в/в в 6-й день }\end{array}$ & Каждые 4 недели \\
\hline Темозоломид & $\begin{array}{l}\text { Темозоломид 150-200 мг/м²/сут. внутрь } \\
\text { в 1-5-й дни }\end{array}$ & Каждые 4 недели \\
\hline $\begin{array}{l}\text { Темозоломид (метрономный } \\
\text { режим) }\end{array}$ & Темозоломид 75 мг/м² внутрь & Ежедневно \\
\hline FOLFOX & 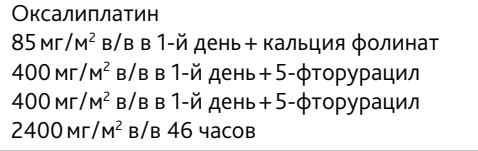 & Каждые 2 недели \\
\hline FOLFIRI & 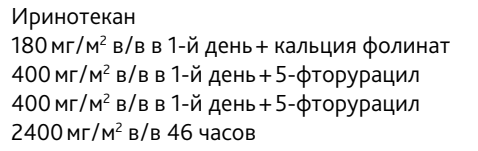 & Каждые 2 недели \\
\hline GEMOX $^{2}$ & $\begin{array}{l}\text { Оксалиплатин } 100 \text { мг/м² в/в в 1-й день +гем- } \\
\text { цитабин } 1000 \text { мг/м² в/в в 1-й и 8-й дни }\end{array}$ & Каждые 3 недели \\
\hline mGEMOX & $\begin{array}{l}\text { Оксалиплатин } 85 \text { мг/м² в/в 1-й день +гемци- } \\
\text { табин } 1000 \text { мг/м² в/в 1-й день }\end{array}$ & Каждые 2 недели \\
\hline $\begin{array}{l}\text { Бевацизумаб +химио- } \\
\text { терапия }^{3}\end{array}$ & $\begin{array}{l}\text { Бевацизумаб 5-7,5 мг/кг в/в в комбинации } \\
\text { с химиотерапией }\end{array}$ & Каждые 2-3 недели \\
\hline
\end{tabular}

1 Не более 3,0 г препарата на курс

2 Режим/nрепарат зарегистрирован в РФ, но не входит в клинические рекомендации, одобренные МинздравомРФ.

${ }^{3}$ Для бевацизумаба не зарегистрировано показание НЭО; в клинических исследованиях применялся в комбинациях с капецитабином.

\subsubsection{2. Химиотерапия нейроэндокринного рака}

Химиотерапия при НЭР является основным методом лечения. В I линии стандартно назначаются режимы на основе препаратов платины (цисплатин/карбоплатин). В случае прогрессирования после I линии, наступившего более чем через 6 мес., рекомендуется реиндукция режима I линии. В случае более раннего прогрессирования во II и после- 
дующих линиях могут быть назначены режимы с включением таксанов, темозоломида, доксорубицина, циклофосфамида, винкалкалоидов, иринотекана, гемцитабина (табл. 6).

Таблица 6. Рекомендуемые режимы химиотерапии нейроэндокринного рака (G3)

\begin{tabular}{|c|c|}
\hline EP & Этопозид 100 мг/м² в/в в 1-3-й дни + цисплатин 75 мг/м² в/в в 1-й день 1 раз в 3 нед., 6 циклов \\
\hline $\mathrm{EC}$ & Этопозид 100 мг/м² в/в в 1-3-й дни + карбоплатин AUC 5 в/в в 1-й день 1 раз в 3 нед., 6 циклов \\
\hline TEMCAP & $\begin{array}{l}\text { Темозоломид } 150 \text { мг/м² внутрь в 10-14-й дни + капецитабин } 2000 \text { мг/м² внутрь в 1-14-й дни } \\
1 \text { раз в } 4 \text { нед., } 6 \text { циклов }\end{array}$ \\
\hline $\begin{array}{l}\text { Карбоплатин+ } \\
\text { иринотекан }\end{array}$ & $\begin{array}{l}\text { Карбоплатин AUC } 5 \text { в/в в 1-й день + иринотекан } 65 \text { мг/м² в/в в 1-й и 8-й дни, каждые } \\
3 \text { недели }\end{array}$ \\
\hline $\begin{array}{l}\text { Цисплатин+ } \\
\text { иринотекан }\end{array}$ & $\begin{array}{l}\text { Цисплатин } 60 \text { мг/м² в/в в 1-й день + иринотекан } 65 \text { мг/м² в/в в 1-й и 8-й дни, каждые } \\
3 \text { недели }\end{array}$ \\
\hline XELOX & $\begin{array}{l}\text { Оксалиплатин } 130 \text { мг/м² в/в в 1-й день + капецитабин } 2000 \text { мг/м² внутрь в 1-14-й дни } 1 \text { раз } \\
\text { в } 3 \text { нед. } 6 \text { циклов }\end{array}$ \\
\hline GEMOX & $\begin{array}{l}\text { Оксалиплатин } 100 \text { мг/м² в/в в 1-й день + гемцитабин } 1000 \text { мг/м² в/в в 1-й день } 1 \text { раз } \\
\text { в } 2 \text { нед., } 9 \text { циклов }\end{array}$ \\
\hline FOLFOX & $\begin{array}{l}\text { Оксалиплатин } 85 \text { мг/м² в/в в 1-й день + кальция фолинат } 400 \text { мг/м² в/в в 1-й день + 5-фтор- } \\
\text { урацил } 400 \text { мг/м² в/в в 1-й день + 5-фторурацил } 2400 \text { мг/м² в/в 46-часовая инфузия } 1 \text { раз } \\
\text { в } 2 \text { нед., } 9 \text { циклов }\end{array}$ \\
\hline FOLFIRI & 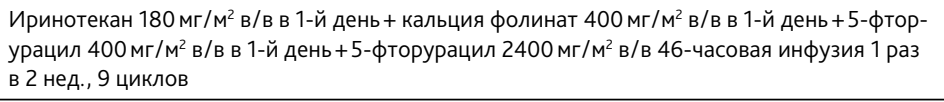 \\
\hline $\begin{array}{l}\text { Эпирубицин+ } \\
\text { дакарбазин+ } \\
\text { 5-фторурацил }\end{array}$ & $\begin{array}{l}\text { Эпирубицин } 25 \text { мг/м² в/в в 1-3-й дни + дакарбазин } 200 \text { мг/м² в/в в 1-3-й дни + 5-фтор- } \\
\text { урацил } 250 \text { мг/м² в/в в 1-3-й дни } 1 \text { раз в } 3 \text { нед., } 6 \text { циклов }\end{array}$ \\
\hline Темозоломид & $\begin{array}{l}\text { Темозоломид 150-200 мг/м²/сут. внутрь в 1-5-й дни } 1 \text { раз в } 4 \text { нед., } 6 \text { циклов. При отсут- } \\
\text { ствии значимой токсичности и нарастании эффекта возможно проведение 8-12 циклов; } \\
\text { для ослабленных больных возможно использовать метрономную терапию темозоломидом } \\
\text { в дозе } 100 \text { мг/сут. или капецитабином по 500 мг 2-3 раза в сутки ежедневно без перерыва } \\
\text { до прогрессирования или непереносимой токсичности }\end{array}$ \\
\hline $\begin{array}{l}\text { Паклитаксел+ } \\
\text { карбоплатин }\end{array}$ & Паклитаксел 175 мг/м² в/в в 1-й день + карбоплатин AUC 5 в/в в 1-й день, каждые 3-4 нед. \\
\hline $\begin{array}{l}\text { Паклитаксел+ } \\
\text { цисплатин }\end{array}$ & Паклитаксел 175 мг/м² в/в в 1-й день + цисплатин 75 мг/м² в/в в 1-й день, каждые 3 недели \\
\hline $\begin{array}{l}\text { Доцетаксел+ } \\
\text { цисплатин }^{1}\end{array}$ & Доцетаксел 75 мг/м² в/в в 1-й день + цисплатин 75 мг/м² в/в в 1-й день, каждые 3 недели \\
\hline $\begin{array}{l}\text { Гемцитабин+ } \\
\text { цисплатин }\end{array}$ & $\begin{array}{l}\text { Гемцитабин } 1000 \text { мг/м² в/в в 1-й, 8-й день + цисплатин } 70 \text { мг/м² в/в в 1-й день, каждые } \\
3 \text { недели }\end{array}$ \\
\hline
\end{tabular}

1 Режим/nрепарат зарегистрирован в РФ, но не входит в клинические рекомендации, одобренные Минздравом РФ.

Лечебные подходы к пациентам с метастазами НЭО не выявленной первичной локализации и внелегочной локализацией мелкоклеточного рака аналогичны таковым для 
нейроэндокринных неоплазий ЖКТ и мелкоклеточного рака легкого (см. соответствующие разделы «Рекомендаций»).

\section{2. Локальное лечение}

\subsection{1. Хирургическое}

При операбельном процессе рекомендуется хирургическое лечение. Однозначных рекомендаций относительно нео-и адъювантного лечения при HEO G1, 2, 3 не существует. При G1 неоадъювантное лечение не проводится, при G2, 3 возможно у отдельных больных. Факторами риска являются метастазы в лимфатических узлах, а также повышение биохимических маркеров. Могут обсуждаться аналоги соматостатина, интерфероны, комбинации XT, не содержащие цисплатин в зависимости от индекса Кі 67. Решение в этих случаях должно приниматься на мультидисциплинарном консилиуме. При неоперабельных НЭО G1 и G2 хирургический метод используется для уменьшения опухолевой массы (циторедуктивные операции), что особенно актуально при гормонопродуцирующих опухолях и может использоваться последовательно или совместно с лекарственной терапией. К другим циторедуктивным методам относятся РЧА, эмболизация и химиоэмболизация метастазов в печени. У большинства больных удается достичь значительного уменьшения проявлений карциноидного синдрома.

\subsection{2. Лучевая терапия}

Дистанционная ЛТ проводится с паллиативной (в т. ч. обезболивающей) целью при НЭО любой степени дифференцировки.

Рекомендуемые алгоритмы лечения НЭО ЖКТ и ПЖ представлены на рис. 1 и 2 соответственно.

\section{4. ЛЕКАРСТВЕННОЕ ЛЕЧЕНИЕ КАРЦИНОМЫ МЕРКЕЛЯ}

При метастатической или местнораспространенной неоперабельной карциноме Меркеля (статус ECOG 0-2) рекомендуется проведение системной терапии.

Стандартом терапии I линии (при отсутствии противопоказаний к лечению) является иммунотерапия с использованием MKA блокаторов PDL1 авелумаб и MKA блокаторов PD1 (пембролизума6, ниволумаб (табл. 7). Наиболее изученным и предпочтительным вариантом выбора из всех представленных является авелумаб.

Всем пациентам на фоне лечения МКА-блокаторами PD1 рекомендуется проводить первоначальную радиологическую оценку ответа на лечение не ранее чем через 12 недель от начала терапии (при отсутствии клинического ухудшения состояния пациента). Повторные исследования проводятся через 8-12 недель (при отсутствии клинического ухудшения состояния пациента).

В качестве терапии II и последующих линий, при неэффективности иммунотерапии МКА блокаторами PD1 и PDL1, вариантом выбора является XT: комбинации EP или EC, а также, 
в качестве схем резерва, могут быть использованы режимы CAV, этопозид или паклитаксел. Карбоплатин используется у ослабленных больных и при нарушении функции почек.

Контрольные обследования для оценки эффективности ХТ прововодят каждые 2-3 цикла.

Пациентам с метастатической и/или неоперабельной карциномой Меркеля рекомендуется также проведение ЛТ с паллиативной целью по показаниям. Показанием при лечении метастатической болезни может быть уменьшение объема опухолевого поражения (в том числе в сочетании с ХT или иммунотерапией) или купирование болевого синдрома.

Проведение иммунотерапии с использованием MKА блокаторов PDL1 (авелумаба) и MKA блокаторов PD1 (пембролизумаба, ниволумаба рекомендовано как в качестве терапии I линии (предпочтительно), так и в качестве терапии II и последующих линий лечения, в тех случаях, когда ранее данный вариант терапии не был использован.

Таблица 7. Режимы лекарственной терапии карциномы Меркеля

\begin{tabular}{|c|c|}
\hline \multicolumn{2}{|c|}{ I линия терапии ${ }^{1}$, а также последующие линии лечения } \\
\hline Авелумаб & 10 мг/кг в/в 60-минутная инфузия каждые 14 дней \\
\hline Пембролизумаб & $\begin{array}{l}2 \text { мг/кг в/в 30-минутная инфузия каждые } 21 \text { день или } 200 \text { мг в/в 30-минутная инфузия } \\
\text { каждые } 21 \text { день }\end{array}$ \\
\hline Ниволумаб & 240 мг в/в 30-минутная инфузия каждые 14 дней \\
\hline \multicolumn{2}{|c|}{ II и последующие линии терапии } \\
\hline EP & $\begin{array}{l}\text { Этопозид } 120 \text { мг/м² в/в в 1-3-й дни + цисплатин 60-80 мг/м² в/в в 1-й день каждые } \\
21 \text { день }\end{array}$ \\
\hline $\mathrm{EC}$ & $\begin{array}{l}\text { Карбоплатин AUC 5-6 в/в в 1-й день + этопозид 100-120 мг/м² в/в в 1-3-й дни каждые } \\
21 \text { день }\end{array}$ \\
\hline CAV & 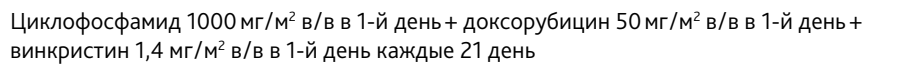 \\
\hline \multicolumn{2}{|l|}{ Схемы резерва } \\
\hline Этопозид & 50 мг/м² внутрь в 1-21-й дни каждые 28 дней \\
\hline Паклитаксел & 80 мг/м² в/в в 1-й, 8-й, 15-й дни каждые 28 дней \\
\hline
\end{tabular}

1 Предпочтительно использование в 1 линии.

\section{5. НАБЛЮДЕНИЕ}

Задачей наблюдения является раннее выявление прогрессирования заболевания с целью своевременного начала лекарственной терапии или проведения хирургического лечения при наличии показаний.

\section{1. Алгоритм наблюдения за больными после операции}

- 1-2-й годы - 1 раз в 3 мес.

- 3-5 годы - 1 раз в 6 мес. 
- После 5 лет - 1 раз в год.

\section{2. Объем обследования на этапе наблюдения и во время лечения}

- Сбор анамнеза и осмотр; осмотр включает пальпацию лимфоузлов регионарных зон, а также выявление симптомов, подозрительных в отношении отдаленных метастазов;

- общий анализ крови с подсчетом лейкоцитарной формулы и количества тромбоцитов;

- биохимический анализ крови с определением показателей функции печени и почек, а также уровня кальция иглюкозы;

- УзИ органов брюшной полости и малого таза, периферических лимфоузлов;

- R-графия органов грудной клетки;

- ЭКГ, ЭхоКГ 1 раз в 6 мес.;

- КТ/МРТ органов брюшной полости и малого таза с в/в контрастированием;

- КТ органов грудной клетки - по показаниям;

- радиоизотопное исследование скелета+R-графия и/или КT/MPT зон накопления РФП - по показаниям;

- биохимические маркеры: кровь - хромогранин А, серотонин, панкреатический полипептид (при НЭО ПЖ, ЖКТ и метастазах НЭО не выявленной первичной локализации); гастрин, глюкагон, инсулин, вазоактивный интестинальный пептид, соматостатин, кальцитонин, АКТГ, кортизол - по показаниям; при карциноидном синдроме - $5-$ ГИУК в суточной моче; ежегодно - NTproBNP; при карциноидной болезни сердца NTproBNP каждые 6 мес.;

- $\quad$ КТ, МРТ головного мозга - по показаниям;

- $\quad$ ПЭТ/КТ - по показаниям (для G2 и G3);

- $\quad$ ПЭТ/КТ с ${ }^{68} \mathrm{Ga}$ - по показаниям (для $\mathrm{G} 1$ и G2).

- $\quad$ ПЭТ/КТ с ${ }^{18} \mathrm{~F}-$ ФДГ - по показаниям (для G2, G3);

- ПЭT/KT с 68DOTA-TATE Ga-DOTA-TOC, DOTA-NOC, (DOTA конъюгаты рецепторов соматостатина) - по показаниям (для G1, G2);

- сцинтиграфия с пентетреодитом и/или ${ }^{99 m T c-Т е к т р о т и д о м п о ~ п о к а з а н и я м ~(д л я ~ G 1, ~ G 2) ; ~}$

- сцинтиграфия $\ln ^{111}$ (октреоскан) - по показаниям (для G1, G2).

Алгоритм наблюдения за больными см. в табл. 7 в разделе «Практические рекомендации по лекарственному лечению нейроэндокринных опухолей легких и тимуса» 

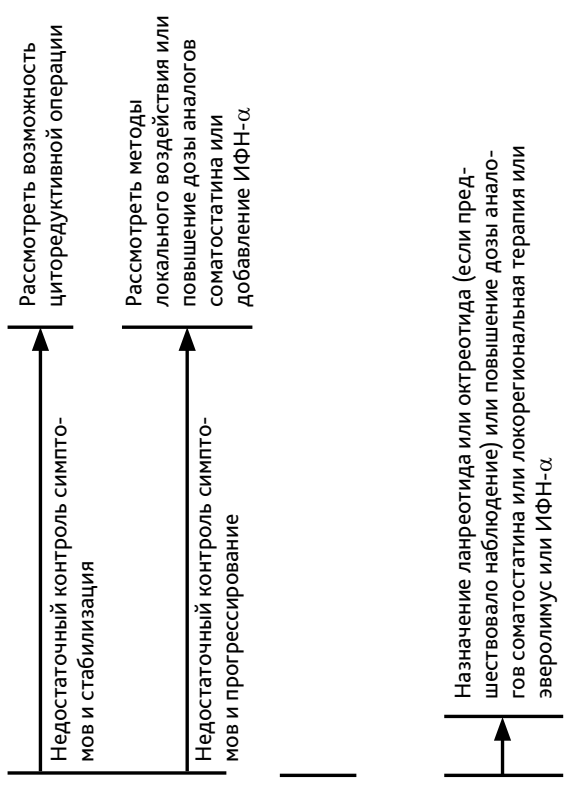

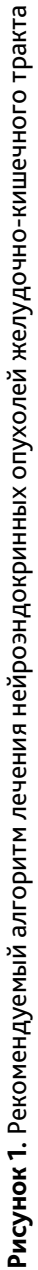
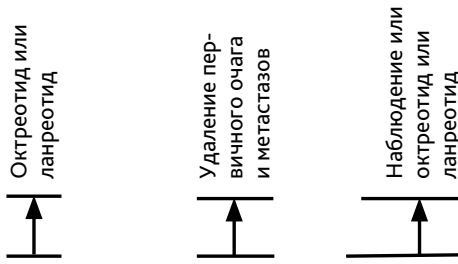

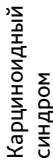
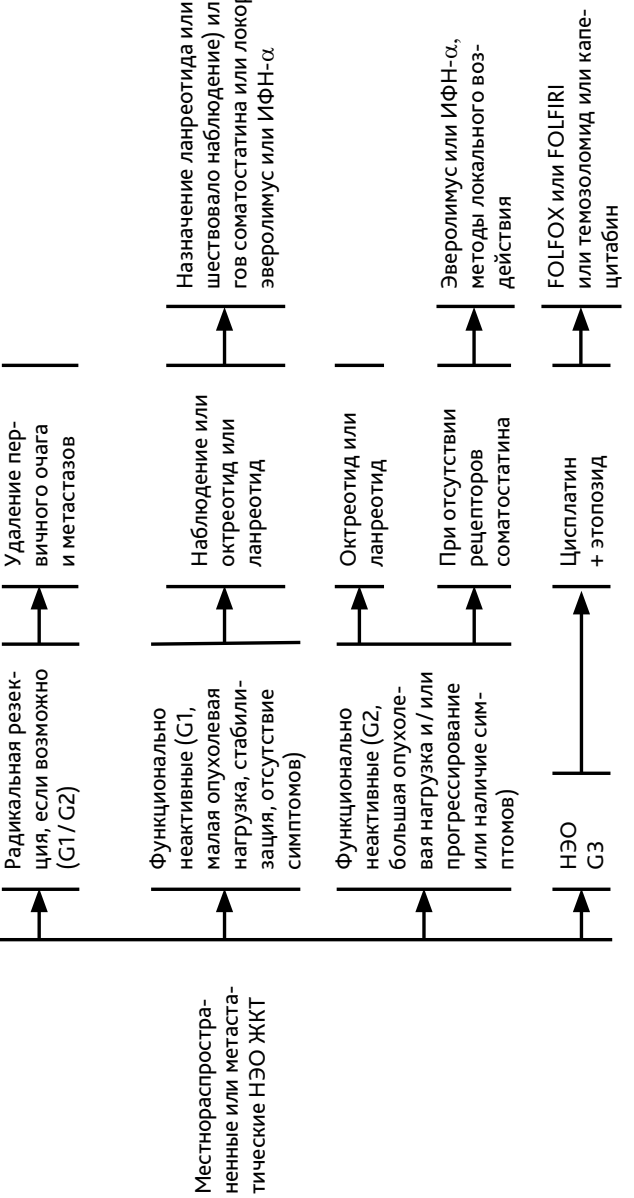

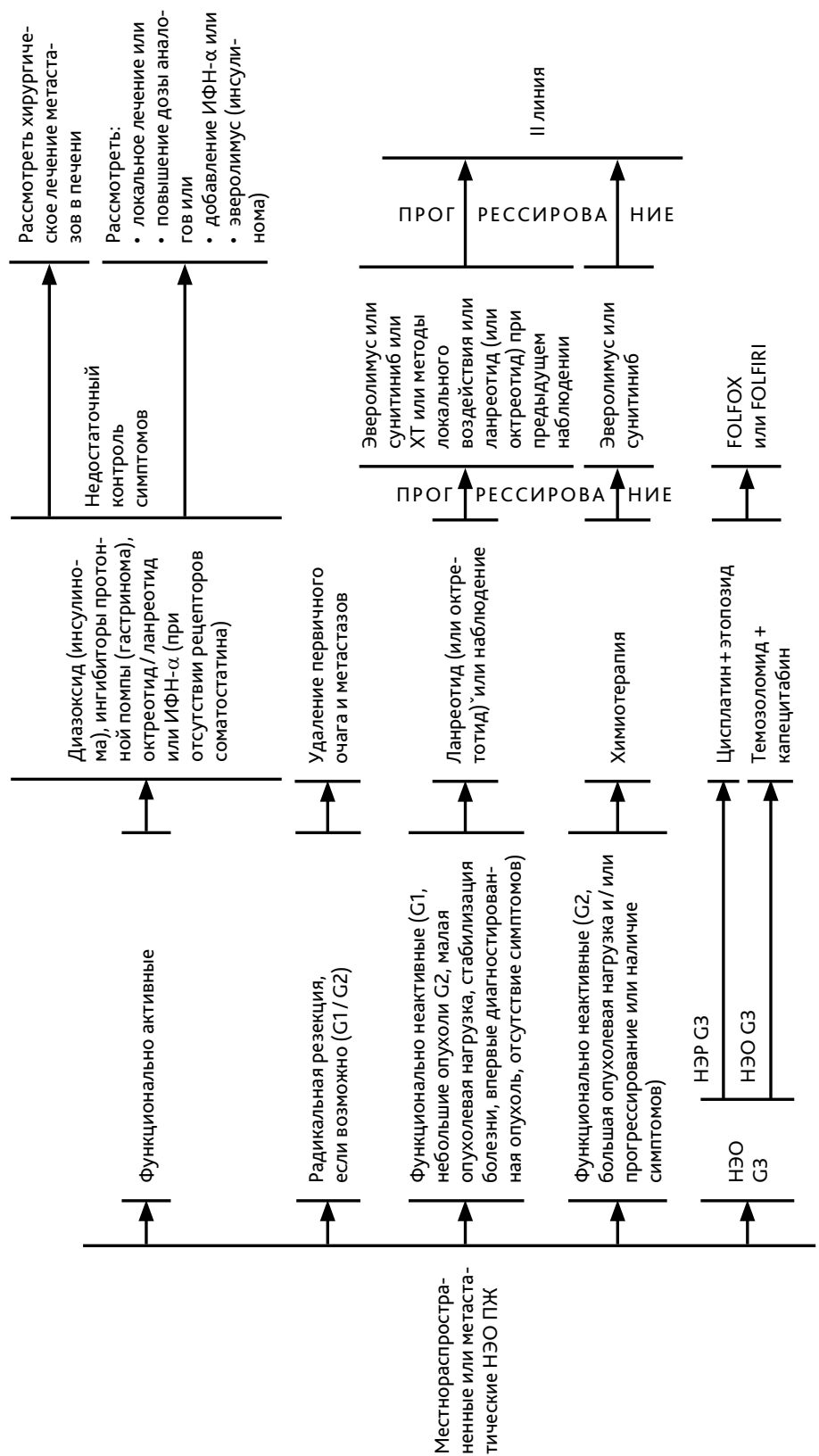

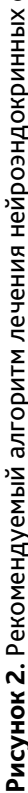


Таблица 9. Рекомендации по использованию маркеров НЭО

(ENETS Consensus Guidelines, 2017 г.)

\begin{tabular}{|c|c|}
\hline Тип НЭО & Маркеры \\
\hline Не функционирующие & $\begin{array}{ll}\text { - } & \text { ХгА } \\
\text { - } & \text { ПП (НЭО ПЖ) } \\
\text { - } & \text { НСЕ (НЭО легких и тимуса G2-3) }\end{array}$ \\
\hline Функционирующие & 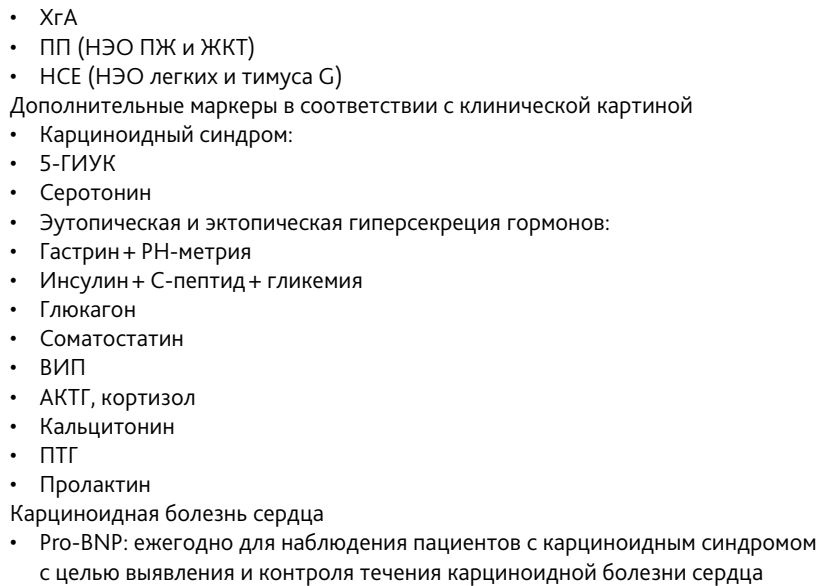 \\
\hline
\end{tabular}

Сокращения: ХгА - хромогранин А, 5-ГИУК - 5-гаммаиндолуксусная кислота, НСЕ-нейронспецифическая енолаза, АКТГ-адренокортикотропный гормон, ПТГ-паратиреоидный гормон, ПП- панкреатический полипептид, ВИП-вазоинтестинальный пептид/ 